\title{
EPITAXIAL LATERAL OVERGROWTH - A TOOL FOR DISLOCATION BLOCKADE IN MULTILAYER SYSTEMS
}

\author{
Z.R. ZYTKIEWICZ* \\ Institute of Physics, Polish Academy of Sciences \\ Al. Lotników 32/46, 02-668 Warsaw, Poland
}

Results on epitaxial lateral overgrowth of GaAs layers are reported. The methods of controlling the growth anisotropy, the effect of substrate defects filtration in epitaxial lateral overgrowth procedure and influence of the mask on properties of epitaxial lateral overgrowth layers will be discussed. The case of GaAs epitaxial lateral overgrowth layers grown by liquid phase epitaxy on heavily dislocated GaAs substrates was chosen as an example to illustrate the processes discussed. The similarities between our results and those reported recently for GaN layers grown laterally by metallorganic vapour phase epitaxy will be underlined.

PACS numbers: 68.55.Ln, 81.15.Lm, 68.60.Bs, 61.72.Ff

\section{Introduction}

Epitaxial lateral overgrowth (ELO) is a method of selective epitaxial growth on partially masked substrates. Prior to growth the substrate is covered by a thin $\mathrm{SiO}_{2}$ or $\mathrm{Si}_{3} \mathrm{~N}_{4}$ film which is next patterned by the standard photolithography. The growth of the ELO layers starts selectively in mask-free seeding areas and proceeds laterally over the dielectric film as shown schematically in Fig. 1. The key advantage of the ELO technique is that substrate defects can propagate to the layer only through a narrow seed, and therefore, a defect density in the layer should be considerably reduced. As a result, the ELO process may be a powerful method to grow epilayers with a low density of dislocations on high dislocation density substrates.

In this work the ways by which the growth rate anisotropy in the ELO technique can be controlled, the effect of substrate defects filtration in the ELO procedure and influence of the mask on properties of the layers will be discussed. A case of the GaAs ELO layers grown by the liquid phase epitaxy (LPE) on heavily dislocated GaAs substrates was chosen as an example to illustrate the processes discussed. However, we will show that the presented phenomena can be observed

\footnotetext{
*e-mail: zytkie@ifpan.edu.pl
} 


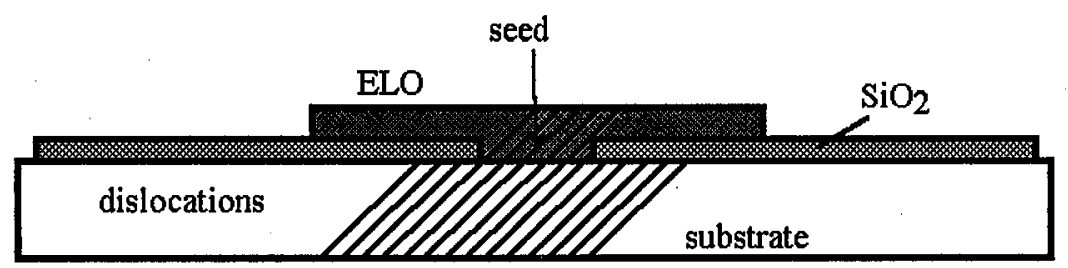

Fig. 1. Schematic drawing of ELO cross-section showing the principle of substrate defects filtration in ELO technique.

for other crystals and growth techniques if they can provide a high selectivity of epitaxial growth. In particular, the recent results on GaN ELO layers grown by metallorganic vapour phase epitaxy (MOVPE) will be referred.

\section{Experimental procedure}

The ELO GaAs layers were grown on (100) GaAs substrates covered by a $0.1 \mu \mathrm{m}$ thick $\mathrm{SiO}_{2}$ film and patterned by a conventional photolithography to form on its whole area a grating of $10 \mu \mathrm{m}$ wide oxide-free line seeding windows spaced by $100 \mu \mathrm{m}$ or $500 \mu \mathrm{m}$. Then, an epitaxial layer was deposited on such substrate by the LPE technique from a $1 \mathrm{~mm}$ thick, saturated Ga-As solution with the use of the piston type, wiping-less graphite boat [1]. The layers were examined by the Nomarski differential interference contrast microscopy (N-DICM), scanning electron microscopy (SEM) and cathodoluminescence (CL) techniques. High resolution X-ray diffraction and synchrotron X-ray topography were used to study the crystallographic quality of the layers.

\section{Results and discussion}

\subsection{Anisotropy of the ELO growth}

It is seen from Fig. 1 that the filtration of substrate defects should be most effective when ELO layers are much wider than the seeds used and for the large values of the ELO width/thickness ratio. Therefore, it is the crucial point of the ELO technique to find the growth conditions under which the lateral growth rate is much larger than that in the vertical direction. The crystallographic orientation of the seed in the plane of the substrate is one of the parameters by varying of which a large ELO growth anisotropy can be forced [2]. The basic idea behind this is to find such orientation of the stripe for which the side walls of the ELO layer are covered by atomically rough, fast growing planes, while the slowly grown facet is formed on the upper surface. We checked this possibility under our growth corrdition by using GaAs substrate with the seeds of star-like pattern on which the GaAs layer was deposited by the LPE [3].

Figure 2 shows the dependence of the lateral growth rate of the GaAs ELO layer versus the orientation of the line seed on the (100) GaAs substrate. It is seen that the lateral growth rate of the ELO was the largest when the seed was $15^{\circ}$ and $30^{\circ}$ off-oriented from the $\langle 011\rangle$ and $\langle 001\rangle$ directions, or their equivalent orientations, while it was the smallest for the $\langle 011\rangle$ and $\langle 001\rangle$ directions. The 


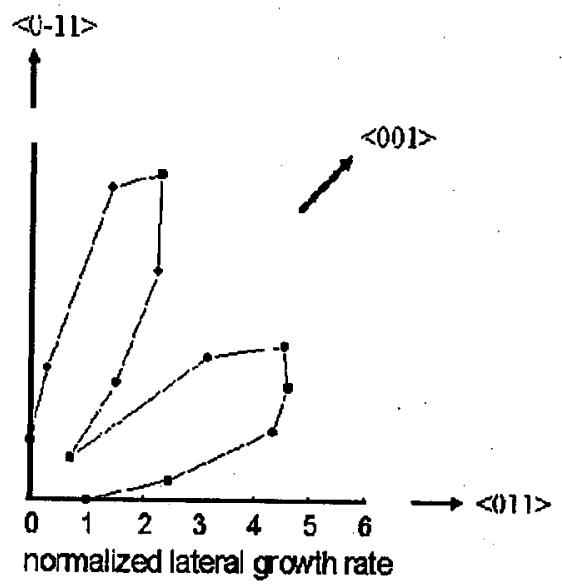

Fig. 2. Lateral growth rate vs. line seed orientation. The growth rate is normalised to its value for the $\langle 011\rangle$ oriented seed.

reason for this was a creation of the slowly grown $\{111\}$ and $\{100\}$ facets on the side walls of the ELO layers. Their formation, which proceeded much faster when the stripe was oriented in the $\langle 011\rangle$ and $\langle 001\rangle$ directions, slowed down the lateral development of the ELO layer. Therefore, on the (100) substrate the line seeds oriented $15^{\circ}, 30^{\circ}, 60^{\circ}$, or $75^{\circ}$ off from the $\langle 011\rangle$ direction should be used to obtain a large value of the lateral to normal growth rate ratio. This finding is in agreement with the results published elsewhere [4]. According to the above discussion, we chose the orientation $75^{\circ}$ off from the $\langle 110\rangle$ direction to grow the ELO layers on the (100) GaAs substrate with the parallel line seeds.

By studying the GaAs ELO growth on misoriented substrates we found that for a low supersaturation at the crystal face the vertical growth rate increases with the density of steps present on the substrate surface [5]. Therefore, the ELO growth rate anisotropy can be low even for the optimal orientation of the seeding line if the density of surface steps in the seeding areas is high. This is a typical situation observed in the relaxed lattice mismatched heteroepitaxial structures in which large number of dislocations supplying surface steps is usually present. Doping has been suggested as an useful tool to force the ELO growth rate anisotropy [6]. Therefore, we have verified this hypothesis to check if the vertical development of ELO caused by a high density of surface steps could be efficiently suppressed by doping [7]. The results obtained are presented in Fig. 3 which shows the values of GaAs ELO width and thickness versus concentration of silicon introduced to the Ga-As solution. For undoped layers isotropic growth was observed. Their thicknesses were comparable to the widths which resulted in the value of the width/thickness ratio being close to 1 . This was due to the high density of surface steps supplied by substrate dislocations as well as by thermal etch pits created during prior to growth annealing of the substrate. However, as shown in Fig. 3, by adding silicon impurity to the melt much thinner and wider layers were obtained. This effect is understood as a result of retardation of surface steps flow on the upper surface of ELO [7, 8]. In such a case the reduction of the growth rate normal to the crystal face is expected for 


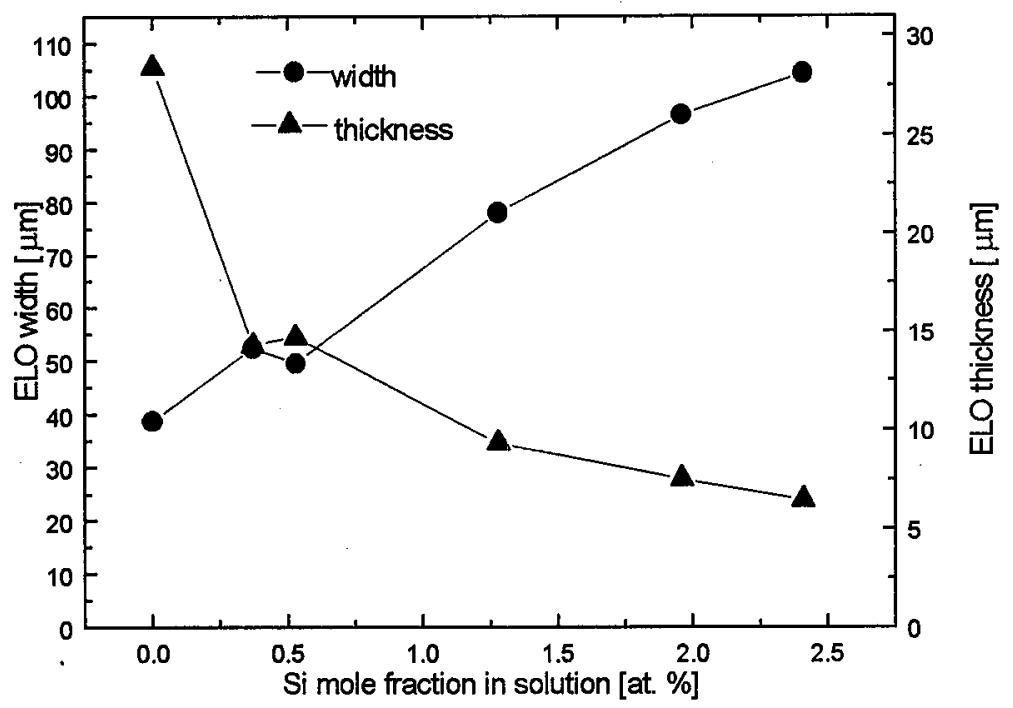

Fig. 3. The width and thickness of LPE grown GaAs ELO layers vs. silicon concentration in the Ga-As melt.

higher doping levels. Due to the fact that incorporation rate of atoms is reduced on the upper surface of ELO the supersaturation in these regions increases during the cooling. Therefore, the diffusion of solute from the upper surface to the side walls must take place. This in turn enhances the lateral growth rate and thinner and wider ELO layers can be grown by increasing the silicon doping, as shown in Fig. 3. It is important to underline that whereas the ELO layers become thinner and wider due to the Si doping the amount of material deposited on the substrate does not change significantly [9]. In this way we have directly proved that impurity atoms affect the redistribution of the growth units on the ELO surface, however they have only a minor influence on the phase equilibrium in the studied system.

Let us mention that the presented results on the LPE growth of GaAs ELO layers on GaAs substrates are a good example of some more general phenomena present during the ELO growth. In particular, a strong dependence of lateral growth rate of MOVPE grown GaN ELO stripes on seed orientation has been reported [10]. Moreover, quite recently magnesium doping during MOVPE of $\mathrm{GaN}$ [11] was found to induce the same changes of ELO growth habit as those obtained by us and shown in Fig. 3.

\subsection{Substrate defects filtration in the ELO procedure}

Figure 4 is a SEM image of the layer grown at $750^{\circ} \mathrm{C}$ by cooling the system with the rate of $0.5^{\circ} \mathrm{C} / \mathrm{min}$ for 40 minutes. The layer was etched after the growth in molten $\mathrm{KOH}$ at $346^{\circ} \mathrm{C}$ for 3 minutes. Such procedure is commonly used to visualise distribution of dislocations in GaAs. First, it is noteworthy that the side walls of the layer are smooth and no low index planes limiting the lateral growth were formed. The main advantage of the ELO technique becomes visible from 
Fig. 4 when the etch pits distributions in the substrate and in the ELO layer are compared. The etch pits density (EPD) in the substrate was high $\left(5 \times 10^{4} \mathrm{~cm}^{-2}\right)$, which was in agreement with the supplier specification of substrates used. On the contrary, in the ELO layers only the dislocations threading from the substrate were observed. As shown in Fig. 4, the density of these dislocations was low and they were confined in a very narrow area above the seed. The rest of the ELO layer was nearly dislocation-free which was additionally proved by the studies of the ELO layers with the use of cathodoluminescence technique as well as by the synchrotron $\mathrm{X}$-ray topography [12]. This finding shows that the mechanism of substrate defects filtration active during the ELO of GaAs was very efficient.

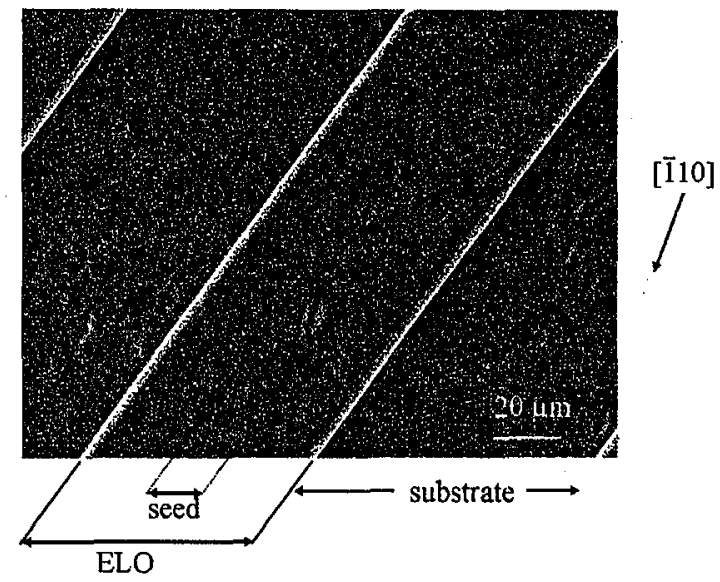

Fig. 4. SEM plane view of ELO structure showing the etch pits distribution in the ELO layer and in the GaAs substrate (from [7]).

There is a number of reports on the substrate dislocations blockade in various epitaxial structures grown by the ELO technique. In particular, the correctness of the schema shown in Fig. 1 has been proved for GaAs ELO layers grown on GaAs [2] or GaAs-coated Si [7] substrates and for GaN ELO layers grown on $\mathrm{GaN}$-coated $\mathrm{SiC}[10,13]$ or GaN-coated sapphire [14] substrates. The latter achievement seems to be the most spectacular one. In fact, the significant reduction of dislocation density in the GaN ELO layers has allowed for the continuous wave operation of blue $\mathrm{GaN}$ laser at room temperature with a lifetime in the 10000 hours range [14].

Let us point out that the structure of substrate dislocations must be taken into account when considering the efficiency of dislocation blockade in the ELO technique. In the GaAs ELO layers grown by LPE on GaAs-coated Si substrates the width of dislocated region above the seed was found to be proportional to the thickness of the layer [6]. This was due to the $60^{\circ}$ type of substrate dislocations being inclined to the substrate surface. Therefore, in such cases the thickness of the ELO layer must be suppressed and its width must be increased to obtain large dislocation-free regions. On the other hand, in the GaN ELO films 
the width/thickness ratio is usually low which is probably due to the larger supersaturation used during the MOVPE growth as well as to the high density of surface steps produced by dislocations in the seeding areas. Despite this the laterally grown parts of GaN ELO layers show a low dislocation density. This success is due in part to the nature of dislocations in GaN which lie mainly parallel to the $c$-axis, which is the growth direction. Therefore, the dislocations originating from the $\mathrm{GaN}$ grown in $\mathrm{SiO}_{2}$ defined trenches did not propagate into the GaN film grown laterally over the $\mathrm{SiO}_{2}$ masked regions and a high quality of ELO layers was obtained.

\subsection{Effect of the mask on properties of the ELO layers}

The sole role of the amorphous mask in the ELO procedure is to provide a barrier for epilayer nucleation on the masked areas of the substrate. Then, the epitaxial growth starts selectively in the seeding areas and the crystallographic structure of the substrate is maintained in the deposited layer. However, only a few results are reported up to now on the interaction between the mask material and the epitaxial layer as well as on the effect of the mask on the quality of ELO structures.

It is well established that the $\mathrm{SiO}_{2}$ mask introduces stress to the substrate on which the oxide film is deposited [15]. This stress may arise from the different thermal expansion coefficients of $\mathrm{SiO}_{2}$ and the substrate or from the volume expansion during the oxide deposition. In fact, with the use of the X-ray diffraction technique we found a tensile strain in the parts of GaAs substrate covered by the $\mathrm{SiO}_{2}$ mask whereas the compressive strain was detected in the oxide-free seeding areas [16]. Accordingly, the deformation of crystal planes in the substrate was the largest at the edges of the mask. This conclusion agrees very well with results on computer simulation of stress distribution in thermally oxidised $\mathrm{Si}$ wafer [17].

Yamaichi et al. [18] have studied the distribution of dislocations in GaAs/Si structures partly covered by $\mathrm{SiO}_{2}$ pattern. They found that dislocations were removed from the oxide-free areas and were collected at the periphery of the $\mathrm{SiO}_{2}$ pattern after the thermal cycling. This finding shows that the localised strain induced by the mask can lead to redistribution of defects in the substrate before any epitaxial growth is started. Thus, the ELO growth may take place from the seeding areas containing a lower density of defects than those present in an unpatterned substrate. The strain induced by the mask can influence the propagation of dislocations from the substrate to the ELO layer, too. Indeed, a bending of dislocations in the vicinity of the $\mathrm{SiO}_{2}$ mask was observed by a transmission electron microscopy in GaN ELO layers [10]. Moreover, new defects can be created at the edge of the mask if stress there becomes too large [19]. This shows that the edges of the mask are the critical points of the epilayer/substrate system and the patterning of the substrate, being an important step of the ELO procedure, must be carefully optimised. In particular, wet etching has been recommended for preparaiion of the oxide-free trenches as it results in a smoothly curved shape of the mask edges [20].

An additional interaction between the closely spaced faces of the mask and of the ELO can be expected when the laterally growing layer starts covering the 
(a)

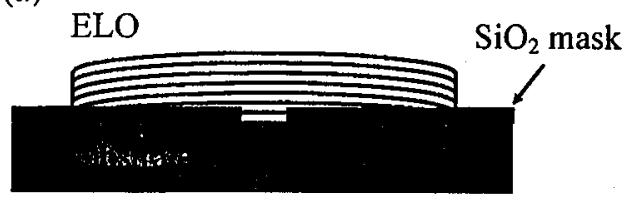

(b)

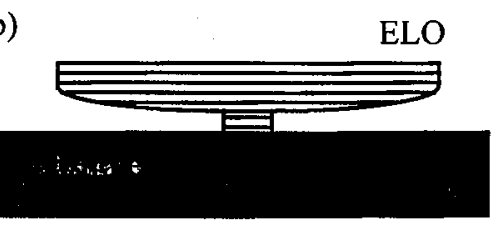

Fig. 5. Schematic drawings of the ELO cross-section. The microscopic bending is shown in the as-grown state (a). The crystal planes of the ELO are undeformed when the $\mathrm{SiO}_{2}$ film is removed by selective etching (b).

oxide film. With the use of high resolution X-ray diffraction [16] and synchrotron $\mathrm{X}$-ray topography [12] techniques we found a pronounced bending of the GaAs ELO layers towards the oxide film. The bending was anisotropic and occurred in the direction perpendicular to the direction of the seeding lines, only. This is schematically shown in Fig. 5a. As a result, broadening of the (400) rocking curves of the as-grown samples was observed under the conditions in which the $\omega$ axis was parallel to the seeding lines. The microscopic bending of the ELO layers disappeared and the rocking curves of flat, unstrained ELO layers were observed when the $\mathrm{SiO}_{2}$ film was removed by selective etching (see Fig. 5b). The similar microscopic bending was recently reported for Si ELO lamellae grown by LPE upon thermally oxidised silicon substrates [21]. The forces resulting from the surface tension of the solution as well as the van der Waals forces attracting the closely spaced surfaces of the ELO and the $\mathrm{SiO}_{2}$ film were suggested as the possible sources of the bending. However, further experimental work is needed for deeper understanding of the sources of this bending and for finding the methods with the use of which the bending could be avoided. In particular, other than $\mathrm{SiO}_{2}$ mask materials must be carefully examined. Graphite might be the suitable mask material. Indeed, our recent experiments indicate that a negligible microscopic bending of the LPE grown GaAs ELO layers is obtained when graphite mask is used to cover the substrate.

Let us mention that the effect of deformation of ELO crystal planes due to the epilayer adhesion to the $\mathrm{SiO}_{2}$ mask has important practical consequences. Large strain can be accumulated and, consequently, new defects can be generated at the seam where two adjacent ELO layers being bent in the opposite directions merge. Indeed, with the use of cathodoluminescence technique we have observed defects created as the result of imperfect coalescence of the ELO layers spreading from adjacent seeds.

\section{Summary}

Results on epitaxial lateral overgrowth of GaAs layers are reported. We have shown that the width/thickness ratio of the ELO layers can be efficiently controlled by optimization of seeds orientation. Moreover, doping has been found to be the way by which the vertical growth rate can be reduced leading to thin and wide ELO layers. All the reports published show that the substrate defects are effectively filtered during the ELO procedure. Therefore, high quality epilayers can be obtained by this growth method despite the high density of defects in 
the substrates used. The question about the possible interactions between the mask and growing ELO layers seems to be still open. However, the mask must be considered as a local source of strain which makes difficult an undisturbed growth of epilayer. Therefore, the use of very thin $\mathrm{SiO}_{2}$ film is recommended with its minimum thickness being determined by the requirement of high growth selectivity. Finally, we have shown that the results obtained are not a specific attribute of the LPE ELO growth of the GaAs layers on the GaAs substrates. On the contrary, the comparison with quite recent reports on the ELO growth of GaN layers by the MOVPE technique shows that our results are a good example of more general phenomena present during the ELO growth of other epitaxial systems.

\section{Acknowledgments}

It is my pleasure to acknowledge D. Dobosz for her contribution in the LPE growth of the samples and E. Kaminska and K. Babska for the excellent photolithography processing of the substrates. This work is partly supported by the Committee for Scientific Research under the grant No. 8 T11B 03010.

\section{References}

[1] Zh.I. Alferov, V.M. Andreev, S.G. Konnikov, V.R. Larionov, B.V.Pushny, Kristall und Technik 11, 18 (1976).

[2] T. Nishinaga, Cryst. Prop. Prep. 31, 92 (1991).

[3] Z.R. Zytkiewicz, D. Dobosz, Acta Phys. Pol. A 92, 1079 (1997).

[4] S. Zhang, T. Nishinaga, J. Cryst. Growth 99, 292 (1990).

[5] Z.R. Zytkiewicz, Cryst. Res. Technol. (1998), in print.

[6] S. Sakawa, T. Nishinaga, Jpn. J. Appl. Phys. 31, L359 (1992).

[7] Z.R. Zytkiewicz, D. Dobosz, in: Heterostructure Epitaxy and Devices, Eds. P. Kordos, J. Novak, Kluwer Acad. Pub., Dordrecht 1998, p. 71.

[8] M. Ohara, R.C. Reid, Modelling Crystal Growth Rates from Solution, Prentice-Hall, Englewoods Cliffs 1973, and references therein.

[9] Z.R. Zytkiewicz, D. Dobosz, M. Pawłowska, unpublished results.

[10] O.-H. Nam, M.D. Bremsen, T.S. Zheleva, R.F. Davis, Appl. Phys. Lett. 71, 2638 (1997).

[11] B. Beaumont, S. Haffouz, P. Gibart, Appl. Phys. Lett. 72, 921 (1998).

[12] R. Rantamaki, T. Tuomi, Z.R. Zytkiewicz, D. Dobosz, P. McNally, J. Phys. D (1998), in print.

[13] T.S. Zheleva, O.-H. Nam, M.D. Bremser, R.F. Davis, Appl. Phys. Lett. 71, 2472 (1997).

[14] S. Nakamura, M. Senoh, S. Nagahama, N. Iwasa, T. Yamada, T. Matsushita, H. Kiyoku, Y. Sugimoto, T. Kozaki, H. Uimemoto, M. Sang, K. Chocho, Jpn. J. Appl. Phys. 37, L309 (1998).

[15] R.J. Jaccodine, W.A. Schlegel, J. Appl. Phys. 37, 2429 (1966).

[16] Z.R. Zytkiewicz, J. Domagała, D. Dobosz, J. Bąk-Misiuk, unpublished results.

[17] M. Karilahti, T. Tumi, M. Taskinen, J. Tulkki, H. Lipsanen, P. McNally, Nuovo Cimento D 19, 181 (1997). 
[18] E. Yamaichi, T. Ueda, Q. Gao, Ch. Yamagishi, M. Akiyama, Jpn. J. Appl. Phys. 33, L1442 (1994).

[19] S. Isomae, J. Appl. Phys. 52, 2782 (1981).

[20] F. Banhart, R. Bergmann, F. Phillipp, E. Bauser, Appl. Phys. A 53, 317 (1991).

[21] H. Raidt, R. Kohler, F. Banhart, B. Jenichen, A. Gutjahr, M. Konuma, I. Silier, E. Bauser, J. Appl. Phys. 80, 4101 (1996). 